\title{
Estado y producción de riesgo: estudio de caso de las comunidades ribereñas de Belén-Iquitos, Perú*
}

\author{
Sharon Gorenstein**
}

* Deseo agradecer al Instituto de Estudios Latinoamericanos Teresa Lozano Long de la Universidad de Texas en Austin por la beca de investigación Lozano Long Summer 2017 que apoyó el desarrollo de mi trabajo de campo. También agradezco a Javier Auyero, Daniel Fridman, Michael Young, Aida Villanueva, Maro Youssef, Aldo Panfichi y Guillermo Rochabrún, cuyos comentarios sobre la forma y el contenido fueron extremadamente útiles.

** Licenciada en Sociología por la Pontificia Universidad Católica del Perú y magíster en Estudios Latinoamericanos del Teresa Lozano Long Institute of Latin American Studies (LLILAS) de la Universidad de Texas en Austin. Docente en la Universidad del Pacífico, y funcionaria pública en el área de Supervisión de la Superintendencia Nacional de Educación Superior (Sunedu). Correo electrónico: sh.gorenstein@gmail.com

Fecha de recepción: 02/04/2019. Fecha de aceptación: 15/09/2019 


\title{
Estado y producción de riesgo: estudio de caso de las comunidades ribereñas de Belén-Iquitos, Perú
}

\section{RESUMEN}

Las políticas de desarrollo, o los proyectos de desarrollo en general, han fallado consistentemente en lograr sus objetivos establecidos al estar basados en una "construcción» del país/comunidad/espacio que tiene poca relación con la cultura local. Sin embargo, aunque los proyectos de desarrollo fracasan, el gobierno logra expandir el campo del poder burocrático del Estado en el espacio que necesita ser «desarrollado». Desde una noción de gubernamentalidad, autoridades socialmente legitimadas interfieren en espacios aislados del desarrollo — institucionalizado desde el Estado- y adjudican, desde su posicionalidad, vicios o virtudes, riesgos o su falta de ellos a las comunidades que buscan atender. De esta manera, el riesgo funciona como un cálculo asociado racionalmente al bienestar, como una forma de representar eventos, como las inundaciones, para que pueda ser gobernado. Al centrarme en el contraste entre las percepciones de riesgo del Estado y de las comunidades fluviales de distrito del Belén, intento contribuir a los recientes debates sobre la evaluación de riesgos del gobierno, como la reorganización y simplificación de la naturaleza para adaptarse a los objetivos de los desarrolladores y de las instituciones públicas y privadas. Analizo e identifico tres dimensiones diferentes de la comprensión del riesgo: mirada del río, nociones de desarrollo e identidad belenina.

Palabras clave: riesgo, gubernamentalidad, desarrollo, comunidades fluviales, Belén, Iquitos, Perú.

\section{State and risk production: a case study from river communities in Belen-Iquitos}

\begin{abstract}
Developmental policies, or development projects in general, have consistently failed to achieve their stated objectives by being based on a "construction» of the country/community/space that bears little relation to local culture. While development projects fail, the government succeeds in expanding the field of bureaucratic state power in the space that needs to be «developed». Seen from a notion of governmentality, socially legitimated authorities interfere in spaces isolated from the actual development by focusing on adjudicating the vices or virtues of developmental policies and their risks or lack thereof. In this way, risk works as a calculative rationally associated with welfare as a way of representing events, such as flooding, so they can be made governable. By focusing on the contrast between the risk perceptions of the state and river communities in the district of Belen, I attempt to contribute to recent debates about government risk assessment as the reorganization and simplification of nature to suit developers' and public and private institutions' goals. I analyze and identify three different dimensions of risk understanding: view of the river; notions of development; and belenino identity.
\end{abstract}

Keywords: risk, governmentality, development, river communities, Belen, Iquitos, Peru. 


\section{INTRODUCCIÓN}

En noviembre de 2014, el gobierno peruano promulgó una ley que declaraba la urgencia de reubicar a las comunidades de Bajo Belén debido a las constantes inundaciones. En abril de 2015, el Ministerio de Vivienda Construcción y Saneamiento (MVCS) financió el primer proyecto de reasentamiento. El propósito, según el gobierno, era brindar a los habitantes de Bajo Belén condiciones de vida más seguras en un espacio con tierra firme. La ley suponía que el proyecto informaría a los beleninos y les daría la libertad de elegir si los reubicaban o no. Según los beleninos, el gobierno no solo no les informó sobre los propósitos reales del proyecto, sino que también utilizó estrategias de intimidación para influir en su toma de decisiones. Además, para los beleninos, el concepto de inundación, utilizado por el gobierno, interpreta erróneamente las fluctuaciones naturales del río que no causan peligro. Al aplicar su propia definición de riesgo, el gobierno diseñó un proyecto de reubicación como una política de evaluación de riesgos que apunta a ganar y mantener su legitimidad y su poder ante la opinión pública (Marlor, 2010). En lugar de aprovechar el conocimiento local como base de sus políticas públicas, el gobierno deslegitimó la cosmovisión de los beleninos sobre las fluctuaciones del río, vistas como un recurso.

¿Cuáles son las dimensiones que explican la diferencia en la comprensión del riesgo de los beleninos y del Estado? Si bien existe una vasta literatura sobre las evaluaciones de riesgo en Perú y el impacto de las reubicaciones, se ha prestado poca atención a los casos en que las percepciones de riesgo no son cuantitativamente diferentes, sino que presentan características cualitativamente diversas. Este artículo argumentará que el contraste entre las percepciones de riesgo del Estado y las comunidades de Bajo Belén no es una mera discrepancia, sino una noción esencial de gubernamentalidad en la cual el contraste en la percepción de riesgo es crucial. A través del proyecto de reasentamiento, como como medida de una acción pública de desarrollo, el gobierno peruano produjo una nueva situación de riesgo.

Según el antropólogo James Ferguson (1994), las políticas de desarrollo —o los proyectos de desarrollo en general — han fallado consistentemente en lograr sus objetivos establecidos al basarse en una "construcción» del país/comunidad/espacio que tiene poca relación con la cultura local. Siguiendo a Ferguson, mientras que los proyectos de desarrollo fracasan al usar estrategias y tácticas para asegurar el «bienestar», el gobierno logra expandir el campo del poder estatal burocrático en el espacio que necesita ser «desarrollado». Visto desde una noción de gubernamentalidad, las autoridades socialmente legitimadas intervienen en espacios aislados del desarrollo, desde el punto de vista del Estado como entidad, al adjudicar vicios o virtudes, riesgos o su falta de ellos, a las comunidades que buscan atender (Ferguson, 1994; Miller y Rose, 1990). De esta manera, el riesgo funciona como un cálculo asociado 
racionalmente con el bienestar como una forma de representar eventos para que puedan ser gobernados. Según Mitchell Dean (1998) y Francois Ewald (1991), "no existe ningún riesgo», en realidad, es una forma de representar o interpretar los eventos para hacerlos gobernables en el campo de las intervenciones políticas (Dean, 1998, p. 25). Por lo tanto, el riesgo en las políticas de desarrollo está continuamente influenciado por las categorías de desarrollo occidentales que visualizan al Estado como una entidad con actores legítimos que pueden ignorar las características esenciales de los órdenes sociales de las culturas locales (Pierce, 2006, p. 897).

Este artículo intenta contribuir a los debates recientes sobre la evaluación de riesgos del gobierno, como la reorganización y simplificación de la naturaleza para adaptarse a los objetivos de los desarrolladores y de las instituciones públicas y privadas. Como demuestra Ulrich Beck (1992, p. 6), el trabajo de campo etnográfico muestra que las opiniones de las personas sobre el riesgo no suelen ser lo que se muestra públicamente. Pueden estar simplemente resignados a la relación que ya tienen con sus instituciones de control. Por lo tanto, la etnografía está mejor preparada para captar situaciones y eventos cotidianos al buscar la inmersión dentro de la comunidad para ver cómo las personas guían sus vidas y para comprender cómo las percepciones de riesgo de las personas se integran en sus rutinas diarias (Heimer, 2001). A través de un estudio etnográfico de diez meses, concurrente con el proyecto de reasentamiento, llegué a entender la realidad de las comunidades de Bajo Belén como algo inaccesible para un planificador de desarrollo (Mitchell, 2009; Babb, 2001; Ferguson, 1994). Complementé la observación participante con entrevistas a profundidad y revisión de documentos para analizar la comprensión del riesgo de los servidores públicos y los expertos en desarrollo.

Este artículo está organizado en tres secciones. La primera presenta el caso de estudio: una comunidad fluvial en la cuenca amazónica del Perú y los orígenes y características del proyecto de reasentamiento. La segunda es una revisión de la literatura que proporciona un marco teórico para comprender las nociones de riesgo y gubernamentalidad y el contraste entre las percepciones de riesgo desde el Estado y desde los beleninos, así como el enfoque metodológico utilizado durante el trabajo de campo. La tercera sección presenta los resultados organizados en tres dimensiones diferentes que explican las discrepancias en la comprensión del riesgo entre los beleninos y el Estado con respecto al Bajo Belén, seguido de una discusión final.

\section{Las comunidades ribereñas de Belén: el caso de estudio}

Belén tiene 68806 habitantes (INEI, 2007) distribuidos en 56 caseríos (pueblos pequeños) y 19 vecindarios en el área urbana. Los principales medios de transporte son pequepeques (canoas) y motocars (motocicletas con asientos adicionales en la 
parte posterior). El puerto de Belén es la principal fuente de comercio y tiene la tasa de criminalidad más alta en todo el distrito. En general, Belén es el ejemplo más extremo de Loreto como región, con indicadores de carácter negativo en términos de salud, educación y violencia (ENDES, 2012).

Los beleninos viven en casas sobre pilotes con pilares de madera que se usan según la temporada. Las casas tienen techos de madera y palma, lo que produce un olor a madera casi mohoso cuando se mezcla con aire caliente. Si bien son rústicos, estos hogares suelen tener sistemas eléctricos y de agua. Durante muchos meses del año, los vecindarios están organizados con puentes de tableros de madera que funcionan como aceras aéreas. Las canoas pasan por debajo de las casas, los niños juegan en el agua contaminada y los buitres de pavos vuelan en círculos en lo alto. Los residuos en el agua incluyen jeringas del centro médico y materia fecal como resultado de los muchos desagües que fluyen hacia el río Itaya. Sin un estudio epidemiológico, se desconoce el grado en que esta contaminación afecta la salud de los habitantes.

La ciudad de Iquitos está aislada debido a la ausencia de infraestructura vial para conectarla con el resto del Perú. Es una ciudad exclusivamente fluvial, lo que significa que la única forma de llegar es por avión o por río. Las principales características de esta ciudad son los ríos de rápido flujo de la cuenca del Amazonas, el clima tropical húmedo y el suelo pobre para la agricultura y la ganadería. De hecho, la interacción entre el clima y el suelo contribuye al movimiento hacia los ríos; en consecuencia, el territorio parece inestable (Vega-Centeno, 2007). La lluvia también da forma a la estructura social y urbana de Belén. Por ejemplo, durante la temporada de lluvias, los beleninos agregan niveles a sus casas para evitar las inundaciones. Mientras que la estación seca o «bajial» entre julio y agosto se caracteriza por una menor precipitación ( $\left.200 \mathrm{ml} / \mathrm{m}^{2}\right)$, durante la "tahuampa» entre enero y marzo, la precipitación puede exceder los $1000 \mathrm{ml} / \mathrm{m}^{2}$, variando el nivel de los ríos en 25 metros e inundando las riberas de los ríos durante cientos de kilómetros (Vega-Centeno, 2007). La variación en las precipitaciones crea una diversidad de espacios geográficos y un ciclo regenerativo para la flora y la fauna. Como Iquitos se encuentra esencialmente en el lecho de un río, es un territorio móvil o ciudad flotante que ha incorporado sistemas urbanos y arquitectónicos flexibles que incluyen complejos residenciales, áreas comerciales y lugares de ocio. Estos sistemas pueden cambiar sus configuraciones físicas para adaptarse a las variaciones en los niveles del río y las inundaciones.

\section{Orígenes del proyecto}

En junio de 2013, el Ministerio de Vivienda, Construcción y Saneamiento (MVCS) y la municipalidad distrital de Belén iniciaron el proyecto «Belén Sostenible». A 
través de la construcción de casas de madera típicas, aceras peatonales y rutas aéreas para vehículos motorizados, el proyecto buscó mejorar el calidad de vida de la población de Belén. La idea era respetar las prácticas culturales locales y mantener un equilibrio ambiental, ecológico y humano. En setiembre de 2013, comenzó la construcción de viviendas. Con el apoyo de la municipalidad distrital de Belén, se construyeron 125 viviendas.

Sin embargo, después de la primera etapa de construcción, el MVCS decidió interrumpir Belén Sostenible y comenzar un nuevo proyecto. Mientras que el primer proyecto consideraba el conocimiento local como la base para su diseńo, respetar las fluctuaciones naturales del río significaba esperar la menor precipitación para comenzar la construcción de las casas. Este período de espera dificultó a los agentes políticos calcular el progreso del proyecto, lo que lo hizo ineficiente. En 2014, el gobierno de Perú declaró a las comunidades fluviales de Belén en estado de emergencia y necesitadas de reubicación debido a la constante inundación del río Itaya. En la ley que apoya el proyecto de reasentamiento, el gobierno peruano propone ideas sobre el riesgo que deslegitima la visión del mundo de los beleninos. Por lo tanto, en noviembre de 2014, la ley 30291 aprobó la declaración de las comunidades de Bajo Belén en riesgo debido a las constantes inundaciones. La estrategia fue reubicar a los beleninos en tierras secas para un mejor y más fácil monitoreo de su «desarrollo». Esta estrategia fue similar a lo que O’Malley (1996) llama «técnicas para intensificar la eficiencia del gobierno" y lo que Dean (1998) y Rose (1996) han observado cuando los expertos en planificación institucional necesitan que sus proyectos sean «auditables».

En febrero de 2015, la Marina de Guerra peruana presentó un informe técnico sobre la variación del curso del río Amazonas en relación con el río Itaya y apoyó el proyecto de reasentamiento. Este indicaba que el río Amazonas volvería a su curso original, siguiendo lo que solía ser su trayectoria en la década de 1700 (el río Itaya) en aproximadamente 51 años, y se apoderaría del río Itaya, inundando así el Bajo Belén (Marina de Guerra, informe técnico, 2015). El 30 de abril de 2015, las autoridades locales y nacionales firmaron un acta intergubernamental que garantizaba el apoyo al proyecto de reasentamiento, respaldado por el estudio de la Marina de Guerra sobre las variaciones de los ríos. El MVCS, en consecuencia, financió el primer proyecto de reasentamiento para trasladar a las personas de una de las comunidades de Belén a tierra firme.

Cuando comenzó el proyecto de reubicación, 197 familias firmaron el documento de "entrega», entregando sus casas en Belén para obtener títulos de propiedad en el área de reasentamiento, Varillalito. Esta área está ubicada en el kilómetro 12.6 de la autopista Iquitos-Nauta, una o dos horas en autobús desde Belén. 


\section{Subjetividad, riesgo y poder}

Variables como la calidad de vida, el bienestar comunitario y la confianza entre los miembros de una comunidad y el gobierno local contribuyen a las percepciones de riesgo (Fatti y Patel, 2013; Ritchie y Gill, 2007). Las personas que viven en áreas con circunstancias peligrosas tienden a expresar niveles más altos de preocupación. Sin embargo, la presencia de eventos peligrosos no supone necesariamente una mayor percepción de riesgo (Prelog y Miller, 2013; Halpern-Felsher, Millstein, Ellen, Adler, Tschann y Biehl, 2001). Aunque las probabilidades y consecuencias de los eventos peligrosos son producidas por procesos físicos y naturales que permiten cuantificar el riesgo, las percepciones de riesgo son inherentemente subjetivas (Slovic, 1999, 2010, 2014). Un riesgo puede existir en la imaginación de las personas y no necesariamente ser experimentado, sino construido colectivamente (Das 1997, 1996). En este contexto, el riesgo no existe "por ahí», independientemente de las mentes y culturas de las personas (Slovic, 2010). El concepto de riesgo está inventado en la sociedad y permite a las personas comprender y hacer frente a los peligros e incertidumbres de la vida. Además, las percepciones de riesgo están integradas en las rutinas diarias y en los procesos regulares que estructuran el pensamiento de las personas (Heimer, 2001). Esto explica por qué incluso cuando los individuos tienen suficiente información sobre una situación o evento peligroso, no necesariamente recordarán las percepciones de riesgo (Slovic, 2010). Cuando el gobierno decide implementar una política de evaluación de riesgos, como el proyecto de reasentamiento, entra en el campo de batalla subjetivo de los significados de riesgo en los que cada parte puede tener diferentes interpretaciones de las fluctuaciones del río.

La principal preocupación planteada por la teoría de la percepción del riesgo es el enfoque cultural que explica que los individuos seleccionan y descartan los peligros según los beneficios que las amenazas puedan impartir a su organización social. Para Kathleen Tierney (2014), existe una mayor probabilidad de que las personas desarrollen un escepticismo extremo sobre el riesgo cuando los peligros pueden afectarlos, al tener pocos recursos, como una forma de enfrentarlos. Según ella, los eventos peligrosos, en muchos casos, articulan grupos que tienen pocos recursos económicos y sociales, con el Estado y la cultura dominante. Luego, «algunos actores e instituciones de élite emplean el poder hegemónico para determinar cómo se enmarcan el riesgo, sus consecuencias y su gestión» (Tierney, 2014, p. 242).

En resumen, como escriben Kahan, Braman, Gastil, Slovic y Mertz (2005), el poder puede configurar las formas en que el riesgo se enmarca y se usa para gobernar. Esto no significa que las personas con menos recursos sean agentes pasivos: desarrollan estrategias para hacer frente y seguir funcionando con sus percepciones de riesgo. En este contexto de estructuración de riesgos, las políticas de evaluación 
de riesgos se convierten en técnicas para intensificar el poder y la eficiencia del gobierno (O’Malley, 1996, p. 190). Además, especialmente en el sector público, el riesgo se convierte en una racionalidad calculadora vinculada a una forma de pensar sobre el campo de la acción política y la intervención gubernamental (Dean, 1997, 1998). En otras palabras, el riesgo funciona como una noción esencial de la gubernamentalidad, en la medida en que los servidores públicos y los «expertos» en desarrollo transforman el riesgo en "gestión de riesgos", haciendo posible que las políticas de evaluación de riesgos se apliquen a los problemas sociales como activos para garantizar su bienestar (Gordon, 1991, pp. 38-39).

Por otro lado, al objetivar ciertos eventos como riesgo, los servidores públicos y los expertos en desarrollo pueden alterar la naturaleza y el significado de las cosas que antes se consideraban comunes y benignas (Scott, 1998, p. 13; Ewald, 1991, p. 200). Además, todo puede ser un riesgo y ser tratado como tal de acuerdo con los principios de los programas de desarrollo (Defert, 1991). En palabras de Beck (1992, p. 4), «los riesgos se definen como las probabilidades de daño físico debido a procesos tecnológicos u otros». Por lo tanto, a los expertos técnicos se les da la legitimidad para definir agendas e imponer premisas limitantes a priori en los discursos de riesgo». En el sector público, especialmente, la objetivación del riesgo funciona como un mecanismo para asegurar la racionalidad de la intervención del gobierno (Marlor, 2010).

\section{La producción del riesgo}

Los funcionarios públicos y los expertos en desarrollo son los representantes de un modelo idealizado de política pública. Beck cuestiona las presentaciones y los procedimientos de los expertos como una defensa perniciosa contra la reflexividad, excluida de las interacciones sociales y políticas entre los expertos, y los grupos sociales sobre el riesgo, debido a la suposición sistemática del conocimiento de laboratorio (Beck, 1992, pp. 4 y 79). Según Beck, los representantes de un modelo idealizado de política pública imputan decisiones, reglas y convenciones, y abren la puerta a una feudalización de la práctica del conocimiento científico, a través de intereses económicos y políticos (Beck, 1992, pp. 156 y 157). Sin embargo, también practican una cierta racionalidad formalizada por el cálculo de probabilidades. «Producen riesgos», hacen aparecer el riesgo. Es característico tener cierto tipo de objetividad, dando a los eventos un tipo de realidad que altera su naturaleza, convirtiéndolos en riesgos (Ewald, 1991, pp. 199 y 200).

Para los funcionarios públicos y los responsables políticos, la comprensión del riesgo es una racionalidad calculadora vinculada al campo de la acción política y la intervención gubernamental (Dean, 1998, p. 25). Desde el punto de vista de la planificación institucional, el gobierno desempeña el papel del regulador racional general 
(Foucault, 2008, p. 146). Para los expertos, los riesgos no están predados: tienen que ser construidos, parecen problemáticos (Miller y Rose, 2008), y deben ser «auditables», en modos de cálculo particulares para agentes políticos (Rose, 1996, p. 55). El concepto de gubernamentalidad, un neologismo acuñado por Foucault, proporciona una visión de la complejidad de las percepciones de riesgo. Según Gordon, la gubernamentalidad «se trata de cómo gobernar» (Gordon, 1991, p. 7). Esto significa que la gubernamentalidad también puede incluir técnicas y prácticas para gobernar a las personas en un nivel de soberanía política sobre una sociedad entera (Gordon, 1991, p. 4). Para la soberanía política, los funcionarios públicos y los responsables políticos deben utilizar la técnica de riesgo: el aseguramiento. El gobierno constituye una institución de aseguramiento que ofrece garantías contra riesgos (Ewald, 1991, pp. 198-200). La paradoja, sin embargo, es que la comprensión del riesgo por parte del gobierno no se corresponde con la de los beneficiarios.

\section{Métodos}

Este estudio se basa en 54 entrevistas a profundidad a habitantes de Belén (veintidós), ex habitantes de Belén que ahora viven en el área de reasentamiento (trece), funcionarios públicos de instituciones relacionadas con el proyecto de reasentamiento (dieciséis) y académicos de Iquitos que siguen el proyecto (tres). Además, se realizó un conjunto de conversaciones informales y trabajo de campo etnográfico durante un período de diez meses; distribuido entre octubre de 2014; febrero, marzo y diciembre de 2015; abril, junio y diciembre de 2016, y mayo, junio y julio de 2017, además del trabajo de archivo, principalmente en Iquitos. La mayoría de las entrevistas en profundidad fueron grabadas en audio, transcritas y codificadas para analizar su contenido. Las entrevistas no grabadas fueron codificadas utilizando las notas tomadas durante la entrevista.

El trabajo etnográfico incluyó observación participante de tareas cotidianas como el lavado de ropa, lavado de platos y limpieza del hogar, que permitió conocer cómo estas actividades están intrínsecamente relacionadas con la lluvia y las percepciones de riesgo. La experiencia de "pasar el rato» en Belén fue indispensable para mi investigación.

Mi trabajo de campo se limitó a Bajo Belén (a lo largo del texto, solo «Belén»), a las comunidades cercanas al puerto y al mercado, que son las únicas incluidas en el proyecto de reasentamiento. Los beleninos que compartieron sus vidas conmigo son todos de limitadas condiciones socioeconómicas, sin educación formal (o solo educación primaria), y son hablantes nativos de espańol. Todas las entrevistas se realizaron en castellano después de explicar a los participantes el propósito del estudio y un acuerdo de confidencialidad verbal que incluye no usar nombres reales para proteger sus identidades. 


\section{Estado y comunidad: visiones contrarias y superpuestas}

El estudio de caso del proyecto de reasentamiento demuestra tres dimensiones diferentes que explican las diferencias en la comprensión del riesgo entre los beleninos y el Estado. Sin embargo, estas diferencias, aun cuando presentadas en sus extremos, se superponen en algunos casos: (i) mirada del río; (ii) nociones de desarrollo que ambos grupos tienen sobre el espacio y las características del Bajo Belén, y (iii) el comportamiento del belenino desde ambas perspectivas (identidad).

\begin{tabular}{llll}
\hline \multicolumn{3}{c}{ Dimensiones de riesgo sobre Bajo Belén } \\
\hline Beleninos & Mirada del río & Nociones de desarrollo & Identidad belenina \\
\cline { 2 - 4 } & Recurso & Pesca & Tradiciones \\
& Sostenibilidad & Mercado & Identidad \\
& Lluvia & Chacra & Organización con la \\
& Fluctuaciones naturales & Redes sociales & naturaleza \\
& del río & Lluvia & Solidaridad \\
& & & Conocimiento local \\
\hline Gobierno & Inundación & Menos contaminación & Inmoral \\
& Necesidad de gestión de & Agua utilizable & Sucio \\
& riesgos & Menos violencia & Desviado \\
& Obstáculo para el & interpersonal & Ignorante \\
& aseguramiento del & Menos violencia urbana & Indisciplinado \\
& gobierno & & \\
& Obstáculo para el & & \\
& desarrollo & & \\
\hline
\end{tabular}

\section{Mirada del río}

La primera dimensión explica por qué vivir en el agua es peligroso para un grupo y no para otro. Según el gobierno, la mayoría de los servidores públicos y los expertos en desarrollo, el riesgo de inundación constituye el problema principal, que presenta la necesidad de una política de evaluación de riesgos. Este grupo alinea su comprensión del riesgo de acuerdo con los principios de los programas de desarrollo (Defert, 1991). De acuerdo con estos principios, las fluctuaciones naturales del río y la lluvia son variables que representan un obstáculo para la capacidad del gobierno para dar a los beleninos aseguramiento y desarrollo. Los funcionarios públicos dicen que Bajo Belén es inhabitable porque las inundaciones impiden la construcción de estructuras de concreto y la instalación de desagües e iluminación. En su opinión, el reasentamiento es más fácil para la gestión de riesgos.

Además, el gobierno cita un estudio realizado por la Marina de Guerra que explica que el río Amazonas inevitablemente inundará el Bajo Belén y destruirá sus 
comunidades. Los siguientes dos extractos de la entrevista resumen el discurso de los servidores públicos sobre cómo vivir en el río no es seguro para las poblaciones. Roberto y Margot trabajan para diferentes instituciones relacionadas con el proyecto, ambas representan uno de los principales argumentos para apoyar el proyecto de reasentamiento:

Roberto: «No puedes vivir allí [Bajo Belén], vivir modificando la casa cada vez que se inunda, no puedes vivir así, es inhumano, no puedes mejorar tu casa porque allí no puedes construir estructuras de concreto, debido al agua, no resiste [su estructura], algunas personas tienen electricidad allí, pero luego hay incendios, nada se puede construir allí.»

Margot: «La ley tiene soporte técnico, un informe del Marina de Guerra que indica que el río Amazonas recuperará su curso anterior. Eso significa que toda la zona de tierras bajas de Belén no solo va a inundarse, sino que estará cubierta por las aguas del río Amazonas y ya no será como una inundación, sería algo permanente.»

En contraposición, para los beleninos, el río constituye un recurso para su supervivencia. Los crecientes y vaciantes [niveles altos y bajos del río] les permiten tener diferentes productos para su consumo. Mientras que durante los niveles más altos del río la pesca es abundante, y se benefician de las frutas que crecen en este período, durante los niveles más bajos del río se benefician de la agricultura. Los cambios en el nivel del río son parte de su realidad cotidiana, sus hábitos comunitarios y familiares, su conocimiento compartido de vivir con y en el río y desde el inicio la historia cultural de Bajo Belén. Para su mayoría, el concepto de inundación del gobierno malinterpreta las fluctuaciones naturales del río. El ambiente amazónico naturalmente cohabita con el agua, que organiza su concepción del espacio alrededor y dentro del agua. Por lo tanto, el agua no solo se considera como parte del contexto local del Bajo Belén, sino también como parte de las prácticas culturales de los beleninos y su supervivencia. El enfoque del gobierno hacia el río y el agua en general no es solo una comprensión diferente del riesgo y los medios económicos; para los beleninos, no contar con los recursos del Bajo Belén amenaza su supervivencia, así como su cultura y desarrollo social y económico. Marta y Eduardo han vivido en Bajo Belén toda su vida y han aprendido a vivir con las fluctuaciones de los ríos. Para ellos, vivir en el río no constituye un riesgo:

Marta: «Promulgaron una ley basada en un estudio realizado por la Marina de Guerra del Perú que dice que esta área desaparecerá; tenemos otro informe que dice lo contrario. Cada año hay inundaciones, pero eso es natural, eso no significa que estemos en peligro. El gobierno debería mejorar la calidad de vida de la población aquí en lugar de querer sacarnos.» 
Eduardo: «Debido a los huaicos [deslizamientos de tierra], es por eso que han comenzado a hablar sobre inundaciones. Aquí tenemos la creciente y vaciante [niveles del río superior e inferior]. Aquí no hay riesgo; lo único que tenemos que hacer es tener cuidado.»

Las opiniones del gobierno y de los beleninos sobre el río se oponen y se basan en diferentes entendimientos de riesgo. Para los funcionarios públicos y los expertos en desarrollo, el riesgo funciona como un cálculo racional asociado con el bienestar (Dean, 1998, p. 25) que, en este estudio de caso, no considera las características esenciales de la cultura local (Pierce, 2006, p. 897). Esta visión está en línea con una manera de pensar sobre el campo de la acción política y la intervención gubernamental (Dean, 1998, p. 25), tal como se presenta en las siguientes líneas.

\section{Nociones de desarrollo}

El gobierno ve a Bajo Belén como un lugar subdesarrollado, contaminado y peligroso, sin acceso a agua potable y drenajes. Los desechos en el agua incluyen diversos desechos de los hogares, materia fecal y jeringas del centro médico. Estos desechos atraen ratas, perros y buitres que transportan más desechos orgánicos. El gobierno también está preocupado por la violencia interpersonal y urbana que ocurre dentro de los vecindarios; en otras palabras, violencia doméstica, robos y venta de drogas. Los siguientes testimonios de Pedro, Ricardo y Gilda, servidores públicos que apoyan el proyecto de reasentamiento, resumen ideas institucionalizadas sobre la suciedad del espacio de Belén:

Pedro: «Las familias viven en contaminación permanente, sin exagerar, sobre la basura [...] Ese ambiente no es saludable; el hacinamiento familiar, es su costumbre, es su estilo, pero ese estilo, esa forma puede modificarse [...] Consideramos que no es un lugar para que los seres humanos se desarrollen completamente.»

Ricardo: "Cuando el río está más alto, ves en la mañana que [mientras] un niño se está lavando los dientes, otro va al baño; todo con la misma agua [...] Es una mentalidad diferente de lo que vivimos aquí [en la ciudad de Iquitos]; lo ven como algo normal [...] Para mejorar la calidad de vida, tenemos que mejorar los valores.»

Gilda: «Belén es como un mercado gigante, puedes encontrar todo, te venden todo: drogas, sexo, niños [...] Allí [Bajo Belén], la gente piensa que golpear a las mujeres es normal, que robar es normal, los niños te dirán que su padre es un ladrón, como si fuera normal, o que su madre es una prostituta [...] Actuando de esa manera, no se mejorarán ellos mismos.»

Los beleninos reconocen y comparten la opinión del gobierno de Belén como un lugar «algo» sucio. Afirman que el Estado debería tener más participación con 
respecto a la recolección de basura y proporcionar más agentes de Policía. Desde su perspectiva, hay una falta de participación municipal en el distrito, un lugar que tiene muchos recursos para el desarrollo social y económico. Destacan la diversidad del espacio geográfico de Belén y el ciclo regenerativo de la Amazonía. En sus mentes, Belén es un lugar en el que nadie se muere de hambre. Siempre hay recursos para sobrevivir: el río, la chacra, el mercado y sus redes sociales. Los habitantes de Belén forman fuertes lazos.

Mirla vino a Bajo Belén cuando era una adolescente. Hoy vende carbón y licor de cańa de azúcar. Ella tiene tres hijas y dos hijos. Hoy todos son adultos, pero ella recuerda cómo los crió en Bajo Belén:

No es que todo esté bien aquí, aquí se pueden mejorar muchas cosas, hay muchos matones, pero como en muchos lugares, no están solo aquí en Belén. Aquí hay muchas personas honestas y trabajadoras que solo quieren vivir bien y que sus familias vivan bien. Otro problema es que el municipio no se involucra mucho, y ahora he escuchado que no podrán hacer nada debido al proyecto de reasentamiento, nos han traicionado, han firmado a favor del proyecto y luego no pueden invertir en el desarrollo de Belén. No entiendo por qué no quieren hacer cosas buenas aquí; hay muchos recursos aquí, dijeron que iban a arreglar el mercado, y todavía nada [...] La gente de Belén compra y vende en el mercado y desde sus hogares, es su forma de vida. Vas a pescar temprano, y vas al mercado a vender, y con eso, tienes dinero para comer y ayudar a tu familia. Aquí, nos apoyamos unos a otros, entre los vecinos, siempre nos apoyamos unos a otros. Aquí crece la raíz de yuca, hay frutos, hay varias cosas que funcionan como recursos para nosotros. Por ejemplo, tengo mi cańa de azúcar, con eso preparo licor y lo vendo desde mi casa y, a veces, en el mercado, también vendo caña de azúcar para comer y su jugo. Para mí, Belén me dio oportunidades que no tenía cuando era pequeña. Antes vivía en la chacra con mis padres, y estaba bien, pero estaba muy lejos. Ya hemos hecho una familia aquí, con los vecinos también, y aquí conocí a mi esposo, he tenido a mis hijos; y poco a poco, hemos ido mejorando, tenemos nuestra casa grande, y mis hijos podían estudiar, yo no podía. Aquí, mi familia y yo creemos que todos los que han venido aquí han podido mejorar. Aquí, ipuedes preguntar a cualquiera! Todos te dirán que viven bien, no en la riqueza, pero no falta nada, no como otros lugares pobres donde a veces no tienes suficiente para comer, aquí no mueres de hambre y siempre obtienes un trabajo o algo para tener dinero. Aquí no viviremos en la riqueza, pero seguimos sobreviviendo e incluso mejorando.

Las nociones de desarrollo del gobierno y de los beleninos se enfrentan, pero a la vez se superponen, y se basan en orígenes diversos. Los beleninos reconocen que Belén tiene varios problemas que necesitan mejorar. Sin embargo, la perspectiva institucionalizada de desarrollo del gobierno le ha permitido determinar estándares de normalidad que eliminan el contexto de vida tradicional e intentar reemplazarlo (Beck, 1992). No es sorprendente que este modo de desarrollo y estandarización se 
base en el riesgo y en el aseguramiento (Ewald, 1991). Algunas personas, espacios o situaciones son menos riesgosas y más fáciles de asegurar.

\section{Identidad belenina}

Desde la perspectiva del gobierno, Belén no es solo un lugar sucio, sino un espacio con prácticas sucias y una cultura desviada. La mayoría de los funcionarios públicos ven a los beleninos solo como sujetos con prácticas inmorales como el robo, el tráfico de drogas, la venta de alcohol, el tráfico de personas y los negocios informales. Además, estos empleados cuestionan la cosmovisión de los beleninos porque al vivir alrededor y con el río, aceptan el riesgo de enfermedades debidas a la contaminación del agua y niveles de vida inferiores. En sus palabras, los beleninos viven en un «ciclo de vida vicioso», por lo tanto, rechazar el proyecto de reasentamiento refleja su ignorancia sobre cómo debe vivir una persona. Clara ilustra algunos de los argumentos utilizados por muchos servidores públicos para apoyar el proyecto de reasentamiento y para deslegitimar la cultura local de los beleninos:

Bueno, hago visitas a domicilio para ver si la gente de Bajo Belén quiere inscribirse para ir a Varillalito, entonces también hay talleres informativos sobre el proyecto, como el uso adecuado del agua, que no saben cómo usar. El agua [...] Belén, desde mi punto de vista, tiene sectores un poco sombríos, me asusta porque la gente está bebiendo, también hay drogas. Tengo la sensación de que las personas en Belén viven en su mundo, en su zona de confort. Para una persona que nunca ha estado en Belén, sería impactante. Usted ve a los niños, mientras que uno se está lavando los dientes, el otro está haciendo sus neccesidades [en la misma agua], y para la madre esto es normal, o ve que los niños no van a la escuela, es una mentalidad y un mundo diferente a lo que vemos, pero para ellos es normal. Por ejemplo, un día estábamos haciendo un censo, llamé a la puerta de una casa y salió una nińa. Le pregunté sobre su padre y su madre, y ella me dijo que no estaban en casa. Ella me dijo que tenía dieciséis años, pero su pareja era adulta. Me sorprende que sus padres lo permitan. Creo que es un círculo vicioso. Realizamos un taller para padres para solicitar permiso para trabajar con sus hijos. Buscamos mejorar la calidad de vida en general. Hay que darles valores, seguridad, respeto por sí mismos, amor, solidaridad, la necesidad de estudiar [...] La gente de Bajo Belén es un poco conformista; no ven más allá, por ejemplo, si ganan treinta soles por día, lo gastan todo en el mismo día, viven en el momento [...] Cuando hay una inundación, es inhabitable; la basura se junta, huele terrible, a veces muere un animal, el sol y todo eso, hay algunos que sufren más que otros, más el drenaje que proviene de la ciudad. Las personas que decidieron dejar Bajo Belén han visto una oportunidad, han comprendido que moverse es mejor.

Desde la perspectiva de los beleninos, sus prácticas reflejan su relación con la naturaleza, su identidad y sus tradiciones. Para ellos, muchos problemas deben 
resolverse en Bajo Belén necesitan tanto su esfuerzo colectivo como el apoyo de la municipalidad. Sin embargo, eso no invalida sus conocimientos locales, tradiciones y cómo viven con la naturaleza. Los siguientes testimonios de Magda y José resumen la perspectiva de los beleninos sobre su modo de vida en Bajo Belén. Ambos nacieron en Bajo Belén y se identifican como beleninos:

Magda: El gobierno quiere reubicarnos donde no hay desarrollo. Aquí están nuestras costumbres, nuestra cultura, nuestro trabajo. La mayoría de la gente trabaja en mercados, puertos, ríos y otros en granjas en áreas cercanas.

José: Sabemos cómo vivimos; sabemos cómo vivir [...] ¿Promulgar una ley contra una población en un lugar habitable? ¿Cómo es que Belén es inhabitable? ¿Entonces soy un animal? Soy un belenino, yo nací aquí; mi padre y mi madre me criaron aquí.

Mientras los beleninos relacionan su socialización con su historia e identidad, los funcionarios públicos utilizan descripciones negativas de los beleninos para reforzar los objetivos de su proyecto de reasentamiento. El gobierno no considera la identidad belenina como una identidad real, sino como una forma de enmarcarlos para demostrar el riesgo de su forma de vida (O’Malley, 1996).

\section{La lucha de percepciones y el desarrollo}

El contraste entre las percepciones diferentes sobre riesgo que tienen el Estado y los beleninos es una noción esencial de gubernamentalidad. El riesgo es una noción que permite a las personas entender y hacer frente a los peligros e incertidumbres de la vida; sin embargo, el riesgo funciona como un indicador de cómo gestionar la función gubernamental de procurar una comprensión asociada con el bienestar. El riesgo probable se convierte en una forma de cuantificar y representar eventos para hacerlos gobernables en el campo de la intervención política. En el caso presentado, los funcionarios públicos hicieron que Bajo Belén fuera «auditable» utilizando la técnica de riesgo: el aseguramiento. El gobierno se establece como una entidad que ofrece garantías contra lo que percibe como riesgo, pero esa percepción está en desacuerdo con la cosmovisión y la cultura local de los beleninos.

Este artículo destaca diferentes entendimientos en tres dimensiones que explican el marcado contraste entre la percepción de riesgo de la mayoría de los beleninos y de la mayoría de los servisores publicos y expertos. La primera dimensión es con respecto a la normalidad y seguridad de vivir en y con el río. La percepción de riesgo del gobierno proviene de los principios de los programas de desarrollo (Defert, 1991), pero no reconoce las características esenciales de la cultura local (Pierce, 2006, p. 897). Por el contrario, para los beleninos, el concepto de riesgo del gobierno con respecto a la inundación interpreta erróneamente las fluctuaciones naturales del río. 
Segundo, Bajo Belén no encaja en la perspectiva institucionalizada de desarrollo del Estado (Beck, 1992); los beleninos son considerados subdesarrollados debido a su contexto de vida tradicional como una comunidad fluvial. Hay una notable diferencia en el concepto de la identidad de los beleninos desde el gobierno y los beleninos. Para los agentes del gobierno, los beleninos están atrapados en un ciclo de vida «vicioso" que les impide alcanzar el concepto institucionalizado de desarrollo — desde el Estado—, reconocer su difícil situación y abrazar el proyecto de reasentamiento.

La percepción del riesgo es inherentemente subjetiva (Slovic, 1999, 2010) y se construye colectivamente (Das, 1996, 1997). Tanto los funcionarios públicos como los expertos en desarrollo, y los beleninos, en muchos casos, han tenido experiencias antagónicas con Bajo Belén, lo que refleja la visión de Heimer (2001) sobre cómo las percepciones de riesgo se integran en las rutinas diarias y los procesos regulares que estructuran el pensamiento de las personas - lo cual explica la posibilidad de superposición de percepciones-. Estas experiencias antagónicas explican por qué la mayoría de beleninos rechaza el proyecto de reasentamiento mientras que la mayoría de servidores públicos lo apoya. La adaptación a las fluctuaciones del río es parte de la vida cotidiana de los beleninos, relación que los servidores públicos ven «desde afuera». En lugar de aprovechar el conocimiento local como la base para el diseño de políticas públicas, el gobierno ha diseñado un proyecto de reubicación como una política de evaluación de riesgos que apunta a ganar y mantener la legitimidad y el poder (Marlor, 2010).

El análisis del caso presentado aquí puede contribuir a los recientes debates sobre la evaluación de riesgos del gobierno como la reorganización y simplificación de la naturaleza para adaptarse a los objetivos de los desarrolladores y de las instituciones públicas y privadas, y frente a la pregunta de Ferguson (1994, p. 181) sobre «qqué se debe hacer?», el concepto de transparencia presentado por Marlor (2010) puede ser la respuesta. En este caso de estudio, después de la primera etapa de construcción, el MVCS decidió interrumpir el proyecto Belén Sostenible y comenzar uno nuevo. Las comunidades de Bajo Belén pasaron de ser consideradas fuentes de conocimiento local a una población en riesgo. Como Dean (1998) y Rose (1996) mencionan en su trabajo en los locales de planificación institucional, el gobierno necesitaba que su proyecto de reasentamiento se «hiciera auditable».

¿Es el desarrollo institucionalizado, como un cálculo asociado racionalmente con el bienestar, una buena razón para excluir las características esenciales de la cultura local de los beleninos? Cualquier respuesta a esta última pregunta debe plantearse con el objetivo de la transparencia, no como una forma de hacer que los proyectos de desarrollo sean auditables. 


\section{REFERENCIAS BIBLIOGRÁFICAS}

Babb, Sarah (2001). Managing Mexico: Economists from Nationalism to Neoliberalism. Princeton: Princeton University Press. https://doi.org/10.1515/9780691187600

Beck, U. (1992). Risk Society: Towards a New Modernity. Londres: Sage.

Das, V. (1996). Language and Body: Transactions in the Construction of Pain. Daedalus, 125(1), Social Suffering, 67-91.

Das, V. (1997). Critical Events an Anthropological Perspective on Contemporary India. Dehli: Oxford University Press.

Dean, M. (1998). Risk, Calculable and Incalculable. Soziale Welt, 49(1), 25-42.

Defert, D. (1991). 'Popular life' and insurantial technology. En G. Burchell, C. Gordon y P. Miller (eds.), The Foucault effect: studies in governmentality: with two lectures by and an interview with Michel Foucault (pp. 211-233). Chicago: University of Chicago Press.

Dickert, Stephan, Daniel Västfjäll, Janet Kleber y Paul Slovic (2012). Valuations of human lives: normative expectations and psychological mechanisms of (ir)rationality. Knowledge, Rationality \& Action, 681-691. https://doi.org/10.1007/ s11229-012-0137-4

Encuesta Demográfica y de Salud Familiar (ENDES) (2012). Recuperado de http://proyectos. inei.gob.pe/web/biblioineipub/bancopub/Est/Lib1075/index.htmlENDES 2012

Ewald, F. (1991). Insurance and Risk. En G. Burchell, C. Gordon y P. Miller (eds.), The Foucault effect: studies in governmentality: with two lectures by and an interview with Michel Foucault (pp. 197-210). Chicago: University of Chicago Press.

Fatti, C. y Patel, Z. (2013). Perceptions and responses to urban flood risk: Implications for climate governance in the South. Applied Geography, 36, 13-22.

Ferguson, J. (1994). The Anti-Politics Machine: Development, Depoliticization, and Bureaucratic Power in Lesotho. Minneapolis: University of Minnesota Press.

Gordon, C. (1991). Governmental rationality: an introduction. En G. Burchell, C. Gordon y P. Miller (eds.), The Foucault effect: studies in governmentality: with two lectures by and an interview with Michel Foucault (pp. 1-52). Chicago: University of Chicago Press.

Halpern-Felsher, B., Millstein, S. G., Ellen, J. M., Adler, N. E., Tschann, J. M. y Biehl, M. (2001). The Role of Behavioral Experience in Judging Risks. Health Psychology, 20(2), 120-126. https://doi.org/10.1037//0278-6133.20.2.120

Heimer, C. A. (2001). Cases and Biographies: An Essay on Routinization and the Nature of Comparison. Annual Review of Sociology, 27, 47-76. https://doi.org/10.1146/ annurev.soc.27.1.47

Instituto Nacional de Estadística e Informática (INEI). 2007. Censos Nacionales 2007: XI de Población y VI de Vivienda. Recuperado de http://censos.inei.gob.pe/cpv2007/ tabulados/

Kahan, D., Braman, D., Gastil, J. Slovic, P. y Mertz, C. K. (2005). Gender, Race, and Risk Perception: The Influence of Cultural Status Anxiety. https://doi.org/10.2139/ ssrn.723762 
Marlor, C. (2010). Bureaucracy, Democracy and Exclusion: Why Indigenous Knowledge Holders Have a Hard Time Being Taken Seriously. Qualitative Sociology (2010), 33, 513-531 https://doi.org/10.1007/s11133-010-9168-7

Miller, P. y Rose, N. (1990). Governing Economic Life. Economy and Society, 19(1), 1-31. https://doi.org/10.1080/03085149000000001

Miller, P. y Rose, N. (2008). Governing the Present: Administering Economic, Social and Personal Life. Introduction: Governing Economic and Social Life (pp. 1-25). Cambridge: Polity.

Mitchell, T. (2009). How Neoliberalism Makes Its World: The Urban Property Rights Project in Peru. En Philip Mirowski y Dieter Plehwe (eds.), The Road from Mont Pelerin: The Making of the Neoliberal Thought Collective (pp. 386-416). Cambridge, MA: Harvard University Press. https://doi.org/10.4159/9780674054264-012

O'Malley, P. (1996). Risk and Responsibility. En A. Barry, T. Osborne y N. Rose (eds.), Foucault and Political Reason: Liberalism, Neo-Liberalism and Rationalities of Government (pp. 189-207). Chicago: University of Chicago Press.

Pierce, S. (2006). Looking like a state: colonialism and the discourse of corruption in Northern Nigeria. Society for comparative study of society and history (pp. 887-914). https://doi. org/10.1017/S0010417506000338

Prelog, A. J. y Miller, L. M. (2013). Perceptions of disaster risk and vulnerability in rural Texas. Journal of Rural Social Sciences, 28(3), 1-31.

Ritchie, L. y Gill, D. (2007). Social capital theory as an integrating theoretical framework in technological disaster research. Sociological Spectrum, 27(1), 103-129.

Rose, N. (1996). Governing Advanced Liberal Democracies. En A. Barry, T. Osborne y N. Rose (eds.), Foucault and political reason: liberalism, neo-liberalism, and rationalities of government (pp. 37-64). Chicago: University of Chicago Press.

Scott, J. C. (1998). Seeing like a State. New Haven y Londres: Yale University Press.

Slovic, P. (1999). Trust, Emotion, Sex, Politics, and Science: Surveying the RiskAssessment Battlefield. Risk Analysis, 19(4), 689-701, August 1999. https://doi. org/10.1111/j.1539-6924.1999.tb00439.x

Slovic, P. (2010). The Psychology of Risk. Saúde Soc. São Paulo, 19(4), 731-747. https://doi. org/10.1590/S0104-12902010000400002

Tierney, K. (2014). The social roots of risk. Stanford: Stanford University Press.

Vega-Centeno, P. (2007). Ciudades, territorio y ecosistemas en el Peru. Iquitos. En Ciudades, territorio y ecosistemas en el Perú (pp. 107-121). Lima: Centro de Investigación de la Arquitectura y la Ciudad, Pontificia Universidad Católica del Perú. 